\title{
Hepatic Artery Thrombosis in Liver Transplantation - Case Report and Literature Review
}

\author{
Catalina Nausica Picu ${ }^{1,2^{*}}$, Florin Botea ${ }^{1,2}$, Doina Hrehoret ${ }^{1}$, Sorin Alexandrescu ${ }^{1}$, Razvan Grigorie ${ }^{1}$, \\ Vladislav Brasoveanu $\mathrm{u}^{1,2}$
}

*Corresponding author: Catalina Nausica Picu

"Titu Maiorescu" University

Medical Doctoral School

Bucharest, Romania

E-mail: nausica_picu@yahoo.com

\author{
1"Dan Setlacec" Center of General Surgery and Liver Transplantation, Fundeni Clinical Institute, \\ Bucharest, Romania \\ ${ }^{2}$ "Titu Maiorescu” University of Medicine, Bucharest, Romania
}

Received: 20.03.2021

Accepted: 22.05.2021

Copyright $\odot$ Celsius Publishing House www.sgo-iasgo.com

\begin{abstract}
We present a case of a 63-year-old male with HCC developed on viral cirrhosis (HVB-HVD) Child C, MELD 15 who was performed a cadaveric orthotopic liver transplantation (marginal graft previous perfused and oxygenated using the Liver Assist Device). After one month, in the context of a febrile episode, the patient was discovered a biliary stenosis associated with biliary fistulae for which he was placed a biliary stent (ERCP). The afterwards abdominal CT revealed absence of the visualization of the graft common hepatic artery and its intrahepatic branches. An arteriography in an emergency manner was performed, with administration of Actylise, and then continued with llomedin and Heparin administration, but with no improved imaging appearance. The patient underwent a liver retransplantation - the arterial anastomose was performed between the donor superior mesenteric artery (due to the anatomical variant: accessory right hepatic artery from superior mesenteric artery) and the recipient infrarenal aorta (using iliac cadaveric arterial graft). 18 months after the retransplantation, the CT scan revealed homogeneous hepatic graft and permeable vascular axis.
\end{abstract}

Key words: hepatic artery thrombosis, interventional arterial dethrombosis, liver retransplantation, arterial anatomical variants, arterial graft

\section{INTRODUCTION}

HAT is the second main cause of liver graft failure after primary nonfunction and is associated with markedly increased morbidity, being the leading cause of graft loss (53\%) and mortality (33\%) during the immediate postoperative period. However, improvements in postoperative care have resulted in a marked reduction of its incidence (1). Early hepatic artery thrombosis (HAT) is defined as a thromboembolic occlusion of the hepatic artery that occurred within 1 month after liver transplantation (2). Early HAT is a dreaded complication after liver transplantation. Initially, symptoms, signs and abnormal laboratory values are absent in eHAT, therefore screening by routine Doppler ultrasound is of utmost importance. If eHAT presents with biliary leakage and sepsis, the prognosis is usually dismal resulting in graft loss (2). 


\section{CASE REPORT (table 1)}

The patient, a 63-year-old male with viral cirrhosis (HVB-HVD) Child C, was diagnosed with hepatocellular carcinoma (HCC) segments VI-VII $(42 / 40 \mathrm{~mm})$ in March 2016, for which he was performed a superselective transarterial chemoembolization (TACE) session on the $31^{\text {st }}$ of March 2016. The evolution was marked by residual tumoral tissue (identified at the control CT) and there was performed a second superselective TACE session on the $28^{\text {th }}$ of July 2016. In March 2017, there were identified at the control CT 2 newly lesions in segments VII-VIII (15 mm; $12 \mathrm{~mm})$, for which the patient was performed a third superselective TACE session on the $27^{\text {th }}$ of March 2017.

On the $20^{\text {th }}$ of November 2017, the patient was performed a cadaveric orthotopic liver transplantation (marginal graft) for HCC beyond Milan criteria - viral cirrhosis (HVB-HVD) Child C - MELD 15; the portal path was previous perfused and oxygenated with the Liver Assist Device. The anastomoses were executed in standard manner: piggyback caval anastomosis (threevein technique), end-to-end portal anastomosis, common hepatic artery-common hepatic artery (endto-end anastomosis, Prolene7/0), choledocho-choledochostomy. A sequential revascularization was done, and the graft was first perfused via the portal vein and then via the hepatic artery. The patient received prophylactic low molecular weight heparin. Postoperative arterial patency was examined by Doppler ultrasound every day during the first week routinely.

The postoperative evolution was favorable in the first month; on the $26^{\text {th }}$ of December, the patient became febrile. There was discovered a biliary stenosis associated with biliary fistulae through aMRI scan - on the $27^{\text {th }}$ of December 2017, the patient was placed a biliary stent (ERCP). The subsequent abdominal ultrasound identified a spastic hepatic artery. The afterwards abdominal CT $\left(29^{\text {th }}\right.$ December 2017) revealed absence of the visualization of the graft common hepatic artery and its intrahepatic branches. An arteriographyin an emergency manner was performed, with administration of Actylise, but with no improved imaging appearance. The Actylise was maintained 4 hours on the femoral sheath, then continued with llomedinand Heparin administration. The abdominal CT scan performed $48 \mathrm{~h}$ after the attempt of the arterial dethrombosis revealed complete thrombosis of the common hepatic artery and its intrahepatic branches, globally unchanged compared with the previous examination (fig. 1). The subsequent evolution is marked by hepatic cytolysis and another febrile

Table 1

\begin{tabular}{|c|c|c|}
\hline $03 / 2016$ & Diagnosis & Hepatocellular carcinoma segments VI-VII (42/40mm) \\
\hline $03 / 2016$ & Treatment & Superselective TACE session - partial response \\
\hline $07 / 2016$ & Treatment & Second superselective TACE session \\
\hline $03 / 2017$ & CT & 2 newly appeared lesions in segments VII-VIII $(15 \mathrm{~mm} ; 12 \mathrm{~mm})$ \\
\hline $03 / 2017$ & Treatment & Third superselective TACE session \\
\hline $20 / 11 / 2017$ & Treatment & $\begin{array}{l}\text { Cadaveric orthotopic liver transplantation (marginal graft previous perfused and } \\
\text { oxygenated with the Liver Assist Device) for HCC beyond Milan criteria - viral } \\
\text { cirrhosis (HVB-HVD) Child C - MELD } 15\end{array}$ \\
\hline \multicolumn{3}{|c|}{ Posttransplantation evolution } \\
\hline $26 / 12 / 2017$ & Clinical presentation & Fever \\
\hline $26 / 12 / 2017$ & $\mathrm{CT}$ & Biliary stenosis suspicion \\
\hline $27 / 12 / 2017$ & MRI & Biliary stenosis associated with biliary fistulae \\
\hline $27 / 12 / 2017$ & ERCP & Biliary stent \\
\hline $29 / 12 / 2017$ & CT & Hepatic artery thrombosis \\
\hline $29 / 12 / 2017$ & Arteriography & $\begin{array}{l}\text { Actylise administration } \\
\text { (maintained } 4 \text { hours on the femoral sheath; no improved imaging appearance) }\end{array}$ \\
\hline $29 / 12 / 2017-6 / 01 / 2018$ & Treatment & llomedin and Heparin \\
\hline $31 / 12 / 2017$ & $\begin{array}{l}\text { CT scan performed } 48 \mathrm{~h} \text { after } \\
\text { the attempt of arterial dethrombosis }\end{array}$ & $\begin{array}{l}\text { Complete thrombosis of the common hepatic artery and its intrahepatic branches, } \\
\text { globally unchanged }\end{array}$ \\
\hline $31 / 12 / 2017$ & Relocation of the patient & \\
\hline $6 / 01 / 2018$ & Treatment & $\begin{array}{l}\text { Liver retransplantation for arterial thrombosis - Donor superior mesenteric artery } \\
\text { (due to anatomical variant: accessory RHA from SMA) - recipient infrarenal aorta } \\
\text { (using iliac cadaveric arterial graft) }\end{array}$ \\
\hline $01 / 2018-06 / 2019$ & $\begin{array}{l}\text { Abdominal ultrasounds } \\
\text { CT scans }\end{array}$ & Homogeneous hepatic graft and permeable vascular axis \\
\hline
\end{tabular}




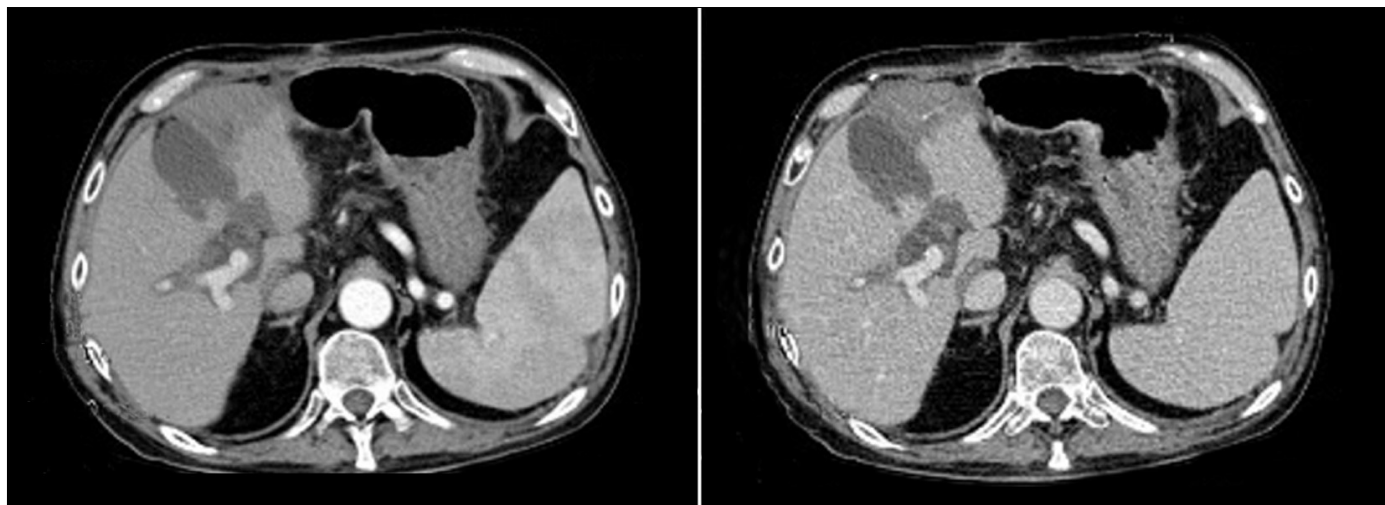

Figure 1 - CT scan performed 48h after attempt of arterial dethrombosis: complete thrombosis of the common hepatic artery and its intrahepatic branches
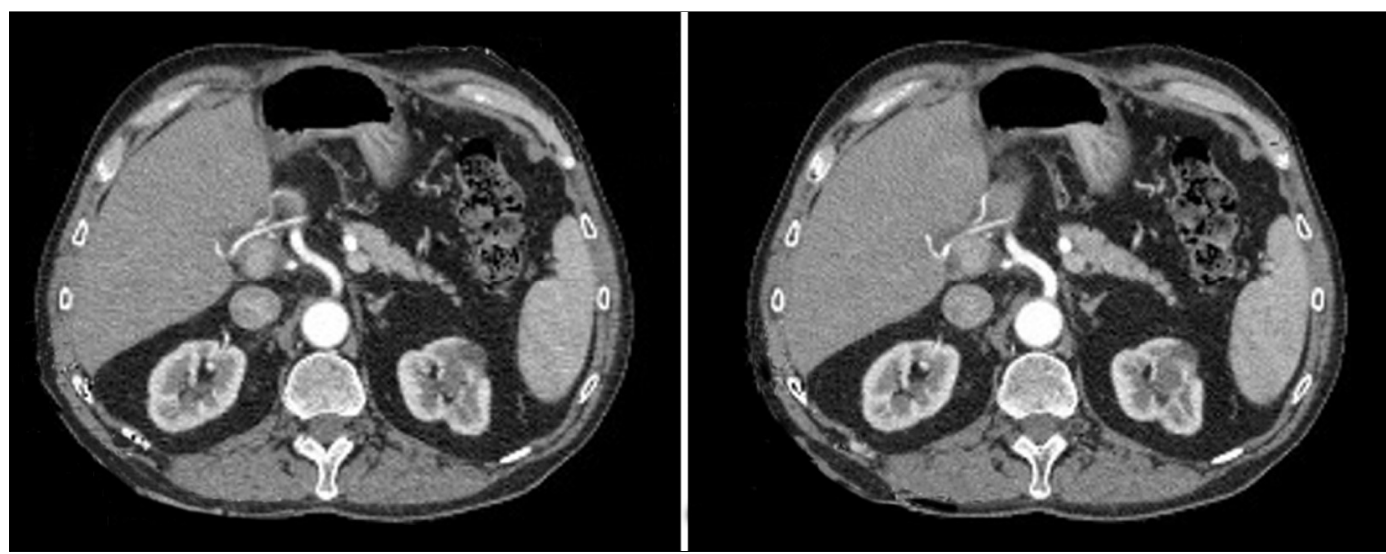

Figure 2 - CT scan after the retransplantation: Homogeneous hepatic graft and permeable vascular axis

episode; the relocation of the patient was decided. On the $6^{\text {th }}$ of January 2018 the patient underwent liver retransplantation for arterial thrombosis. The arterial anastomose was performed between the donor superior mesenteric artery (due to the anatomical variant: accessory right hepatic artery from superior mesenteric artery) and the recipient infrarenal aorta (using iliac cadaveric arterial graft). Re-evaluation abdominal ultrasounds and CT scans until 06/2019 (18 months after the retransplantation) revealed homo-geneous hepatic graft and permeable vascular axis (fig. 2).

\section{DISCUSSION}

HAT represents one important cause of mortality after liver transplantation and is usually divided into 2 categories: early (occurring in less than 1 month after orthotopic liver transplantation) and late (occurring more than 1 month after OLT). The incidence of HAT varies from $2.5 \%$ to $15 \%$. Early HAT is associated with an aggressive course, whereas late HAT has a relatively mild clinical course. Many therapeutic options for managing HAT are available: arterial reconstruction (revision of anastomosis or interposition of an iliac conduit), surgical thrombectomy, radiologically guided thrombolysis with or without angioplasty or stenting, retransplantation (3-5) (fig. 3).

Because the hepatic artery is the only route by which oxygenated blood is supplied to the transplanted liver, obstruction of the hepatic artery results in parenchymal and biliary ischemia. The clinical presentation of hepatic artery thrombosis or stenosis varies from slight signs of parenchymal involvement to fulminating hepatic failure. Early diagnosis and prompt treatment of vascular complications result in longer graft and patient survival.

The incidence of retransplantation varies between $5 \%$ and $22 \%$ worldwide. Analysis of theindications for retransplantation revealed that hepatic artery thrombosis (HAT) was the most common indication for retransplantation, followed by chronic rejection and recurrent disease (6).

The clinical expression depends on the timing of 


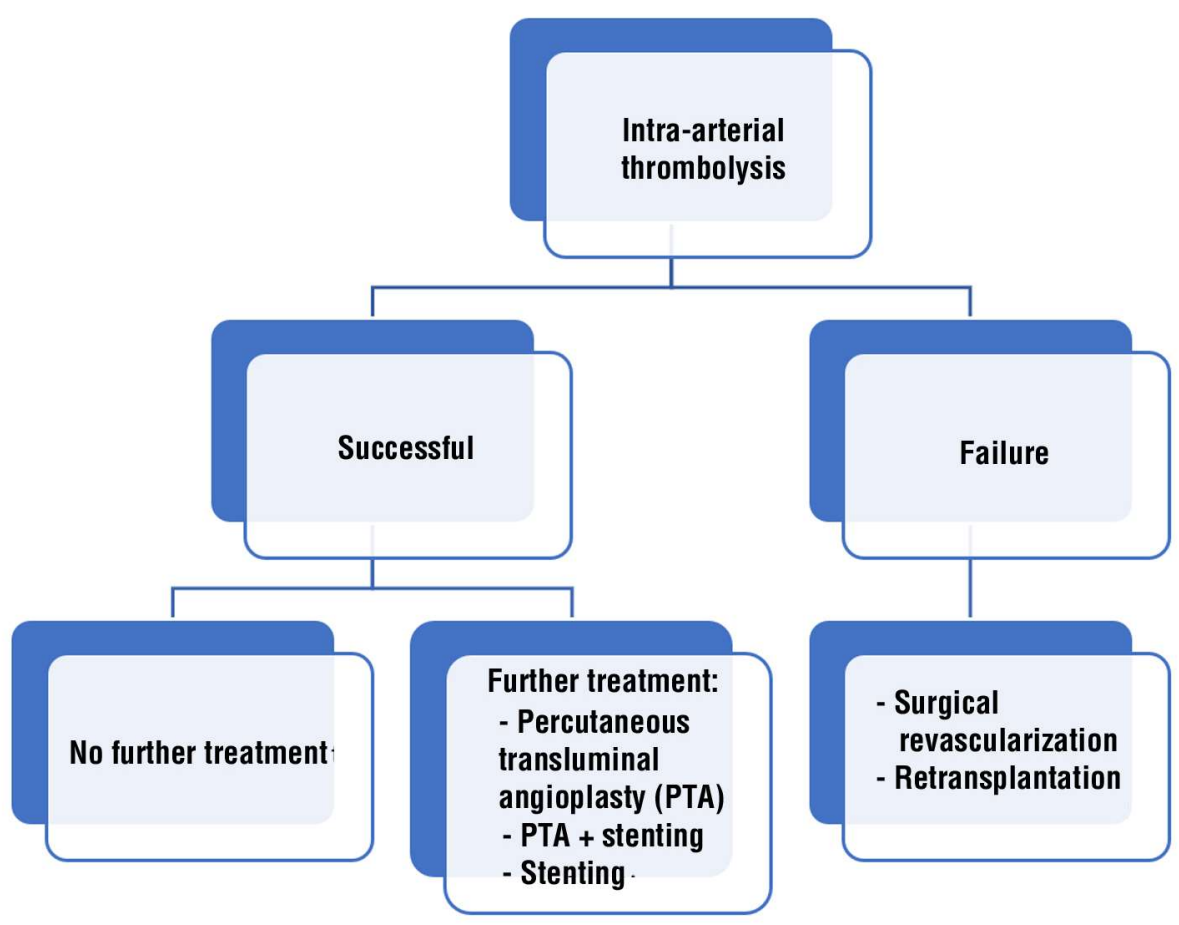

Figure 3 - Hepatic artery thrombosis treatment (5)

the onset of HAT as well as on the existence of collaterals. Late HAT can be asymptomatic, but in most cases the clinical expressions are biliary tract complications such as necrosis and abscess, as well as liver graft ischemia (7).

The key point in vascular complications is early diagnosis, which allows immediate treatment to avoid graft loss. Doppler ultrasonography is the gold standard for screening protocols, because for it detects the absence of hepatic artery flow, even in its intrahepatic branches. The screening protocols of early HAT between different centers are highly variable with respect to frequency. We have established a screening protocol of early HAT, consisting of Doppler ultrasound every day during the first week routinely $(3,5,8)$.

Jonathan G. Stine et al. presented in a large study published in 2015 in HPB journal the fact that pretransplant portal vein thrombosis is associated with posttransplant hepatic artery thrombosis independent of other factors and recipients with portal vein thrombosis might benefit from aggressive coagulation management and careful donor selection. Data on all LTs occurring in the United States between February 2002 and September 2014 were obtained from the Organ and Transplantation Network (OPTN) to analyze HAT in the liver transplant population
(63,182 recipients). Multivariable models were constructed to assess statistical associations and risk factors for the development of HAT. The study documented an ndependent cross-sectional association showing increased risk of early HAT with resultant graft loss in LT recipients with pre-transplant portal vein thrombosis (9).

MA Silva et al. concluded on a ten-year, 1257 liver transplant database from the Liver Unit, Queen Elizabeth Hospital, University Hospital Birmingham that there was no statistical difference in recipient survival between those who developed early vs. late HAT (10).

\section{CONCLUSIONS}

Liver retransplantationis an effective treatment modality for patients with arterial thrombosis and offers good longterm survival $(6,11)$.

The most common indication for retransplantation is early HAT (30.6\%). Nearly two-thirds of the regrafted patients undergo the procedure within 6 months of their primary transplantation. Most of the late retrans- plantations are performed within 5 years of primary transplantation and only $10.7 \%$ of patients require retransplantation after 5 years. Early retransplantationis associated with worse outcome compared with late retransplantation (6). 
K Mohkamet al. revealed (on a 514 OLT recipients study performed between 2005 and 2015 at the CroixRousse University Hospital, Lyon, France) the use of oral systemic vasodilatators (Amlodipine and Nicorandil) combined with systematic control DUS at 2 days as an effective strategy for the management of Doppler ultra- sound arterial abdnormalities (defined by HA resistive index $<0.5$ ) after OLT (12). In addition to this, antiplatelet prophylaxis was found to effectively reduce the incidence of late HAT after LT, particularly in the patients at risk for this complication (13).

Although technical reasons commonly account for HAT, it is also known to be associated with older donors (influencing the occurrence of atherosclerotic lesions in arterial vasculature) and marginal donors $(3,14)$

As reviewed in the literature, other risk factors described to predispose to HAT are: prolonged cold ischemia time greater than $12 \mathrm{~h}$, arterial reconstruction, including the use of a cadaveric iliac jump graft, suture type, small vessel size, ongoing endothelial dysfunction, continued mileu of hypercoagulability (low levels of antithrombin III and high levels of factor VIII), cytomegalovirus (CMV) infection. On the other hand, heparin infusions at cross clamp were associated with a decreased risk of HAT on univariate and multivariable analysis $(4,9)$.

Tzakiset al described three types of presentation (fulminant hepatic necrosis, biliary leak, and relapsing bacteremia) if HAT occurred within 2 months after OLT. Late HAT has been reported to cause biliary tree necrosis, biliary leak, intrahepatic biloma, or abscesses. Early intervention followed by early re-OLT can salvage patients (15).

\section{Conflict of interest}

The authors declare no conflicts of interest.

\section{Ethical approval}

The case was approved by the Ethics Committee.

\section{REFERENCES}

1. Pareja E, Cortes M, Navarro R, Sanjuan F, López R, Mir J. Vascular complications after orthotopic liver transplantation: Hepatic artery thrombosis. Transplantation Proceedings. 2010;42(8):2970-2.

2. Bekker J, Ploem S, de Jong KP. Early hepatic artery thrombosis after liver transplantation: A systematic review of the incidence, outcome and risk factors. American Journal of Transplantation. 2009;9(4): 746-57.

3. Mourad MM, Liossis C, Gunson BK, Mergental H, Isaac J, Muiesan $P$, et al. Etiology and management of hepatic artery thrombosis after adult liver transplantation. Liver Transplantation. 2014;20(6): 713-23.

4. Warner P, Fusai G, Glantzounis GK, Sabin CA, Rolando N, Patch D, et al. Risk factors associated with early hepatic artery thrombosis after orthotopic liver transplantation - Univariable and multivariable analysis. Transplant International. 2011;24(4):401-8.

5. Singhal A, Stokes K, Sebastian A, Wright HI, Kohli V. Endovascular treatment of hepatic artery thrombosis following liver transplantation. Vol. 23, Transplant International. 2010. p. 245-56.

6. Marudanayagam R, Shanmugam V, Sandhu B, Gunson BK, Mirza DF, Mayer D, et al. Liver retransplantation in adults: A single-centre, 25-year experience. HPB. 2010;12(3):217-24.

7. Piardi T, Lhuaire M, Bruno 0, Memeo R, Pessaux P, Kianmanesh R, et al. Vascular complications following liver transplantation: A literature review of advances in 2015. World Journal of Hepatology. 2016;8(1):36-57.

8. Vivarelli M, Cucchetti A, La Barba G, Bellusci R, de Vivo A, Nardo B et al. Ischemic arterial complications after liver transplantation in the adult: multivariate analysis of risk factors. Arch Surg. 2004;139(10): 1069-74

9. Stine JG, Pelletier SJ, Schmitt TM, Porte RJ, Northup PG. Pretransplant portal vein thrombosis is an independent risk factor for graft loss due to hepatic artery thrombosis in liver transplant recipients. HPB. 2016;18(3):279-86.

10. Silva MA, Jambulingam PS, Gunson BK, Mayer D, Buckels JAC, Mirza DF, et al. Hepatic artery thrombosis following orthotopic liver transplantation: A 10-year experience from a single centre in the United Kingdom. Liver Transplantation. 2006 Ja;12(1):146-51.

11. Sugawara $\mathrm{Y}$, Kaneko J, Akamatsu N, Imamura $\mathrm{H}$, Kokudo $\mathrm{N}$, Makuuchi M. Anticoagulant therapy against hepatic artery thrombosis in living donor liver transplantation. Transplant Proc. 2002;34(8):3325-6.

12. Mohkam K, Fanget F, Darnis B, Harbaoui B, Rode A, Charpiat B, et al. Use of systemic vasodilators for the management of Doppler ultrasound arterial abnormalities after orthotopic liver transplantation. Transplantation. 2016;100(12):2671-81.

13. Vivarelli M, la Barba G, Cucchetti A, Lauro A, del Gaudio M, Ravaioli $M$, et al. Can antiplatelet prophylaxis reduce the incidence of hepatic artery thrombosis after liver transplantation? Liver Transplantation. 2007;13(5):651-4.

14. CK Oh, SJ Pelletier, RG Sawyer, AR Dacus et al. Uni- and multivariate analysis of risk factors for early and late hepatic artery thrombosis after liver transplantation. Transplantation. 2001:71:767-772.

15. Gunsar F, Rolando N, Pastacaldi S, Patch D, Raimondo ML, Davidson B, et al. Late hepatic artery thrombosis after orthotopic liver transplantation. Liver Transplantation. 2003;9(6):605-11. 